\title{
Estudiantes transnacionales y calidad de los sistemas educativos en la región Tijuana-San Diego
}

\section{Transnational Students and Quality in Education System in the Tijuana-San Diego Region}

\author{
José Candelario Osuna García \\ Universidad Autónoma de Baja California \\ Tijuana, Baja California, México \\ josuna7@uabc.edu.mx \\ Jocelyne Rabelo Ramirez ${ }^{2}$ \\ Universidad Autónoma de Baja California \\ Tijuana, Baja California, México \\ jrabelo@uabc.edu.mx
}

Recibido: 16/02/2018 Aceptado: 01/06/2018

Resumen. La región Tijuana-San Diego es el espacio binacional más importante de la frontera entre México y Estados Unidos, principalmente como consecuencia del dinamismo e interacción socioeconómica observada, caracterizado por un elevado crecimiento urbano, una fuerte interacción de flujos de capital, trabajadores y mercancías, que promueven la expansión económica y demográfica de la misma región (Alegría, 2010). Sin embargo, la relación entre las dos ciudades también se distingue por compartir grupos de estudiantes que transitan entre dos sistemas educativos con profundas desigualdades en la calidad de la enseñanza, infraestructura y aprendizajes. Situaciones que representan significativos retos en lo que a planeación transfronteriza se refiere (Guillén, 1993).

\footnotetext{
Profesor de la Facultad de Ciencias Humanas de la Universidad Autónoma de Baja California, México. Correo electrónico: josuna7@uabc.edu.mx

2 Profesora de la Facultad de Economía y Relaciones Internacionales de la Universidad Autónoma de Baja California, México. Correo electrónico: jrabelo@uabc.edu.mx
} 
Revista UniversidAd en DiÁlogo • Vol. 9, N. ${ }^{\circ}$ 1, Enero-Junio, 2019 • 63-85

ISSN 2215-2849 • EISSN: 2215-4752

URL: http://www.revistas.una.ac.cr/index.php/dialogo/index CORREO ELECTRÓNICO: universidadendialogo@una.cr

DOI: https://doi.org/10.15359/udre.9-1.4

En esta región, el problema educativo de los estudiantes transnacionales y la desigualdad en el dominio y demostración de los aprendizajes universales son dos fenómenos pedagógicos que cristalizan la urgente necesidad de eliminar las barreras fronterizas entre México y Estados Unidos para intervenir conjuntamente y encontrar una solución. De ahí que el objetivo del presente texto académico consista en describir el panorama actual de la calidad de los aprendizajes en los sistemas escolares de California y Baja California, mediante la comparación de los resultados alcanzados en las evaluaciones internacionales a gran escala, para identificar las implicaciones que tiene este horizonte educativo en la adaptación de los estudiantes transnacionales.

Palabras clave: calidad educativa, estudiantes transnacionales, región transfronteriza.

\begin{abstract}
The Tijuana-San Diego region is the most important binational area on the border between Mexico and the United States, because of the dynamism and observed social-economic interaction, with a high rate of urban growth, a strong interaction of capital flows, workers and goods, which promote the economic and demographic expansion of the same region (Alegría, 2010). However, the relationship between the two countries is also characterized by sharing groups of students that attend two education systems with profound inequalities in regards to the quality of education, infrastructure, and learning. Situations that represent significant challenges in terms of cross-border planning (Guillén López, 1993). In this region, the educational problem of transnational students and the inequality in knowledge and demonstration of universal learning are two pedagogical phenomena that crystallize the urgent need to eliminate the border barriers between Mexico and the United States to carry out actions jointly and find a solution. Therefore, the objective of this academic text is to describe the current landscape of the quality of learning in the school systems of California and Baja California, by comparing the results achieved in large-scale international evaluations, to identify the implications of this educational horizon for the adaptation of transnational students.
\end{abstract}

Keywords: Education quality; transnational students; cross-border region.

\title{
Región transfronteriza y estudiantes migrantes
}

La región fronteriza entre México y Estados Unidos se creó oficialmente con la promulgación del Acuerdo de La Paz en la década de 1980. En él se delimitó como "área fronteriza" a la zona situada dentro de los cien kilómetros con respecto a los límites territoriales y marítimos de ambos países (Acuerdo de La Paz, 1984). En la actualidad, dicha área abarca 48 condados estadounidenses en cuatro estados: California, Arizona, Nuevo México y Texas, así como 94 municipios mexicanos en seis entidades federativas fronterizas: Baja 
URL: http://www.revistas.una.ac.cr/index.php/dialogo/index

CORREO ELECTRÓNICO: universidadendialogo@una.cr

DOI: https://doi.org/10.15359/udre.9-1.4

California, Sonora, Chihuahua, Coahuila, Nuevo León y Tamaulipas (Centro de Estudios Internacionales Gilberto Bosques, 2017).

Esta área es considerada como una región que, en términos económicos, políticos y demográficos, presenta características únicas (Weeks, 1993), entre otras cuestiones, por la integración económica y social consolidada de las concentraciones urbanas binacionales (como es el caso de Tijuana-San Diego, Mexicali-Calexico, Ciudad Juárez-El Paso, Reynosa-McAllen, MatamorosBrownsville, etcétera), así como por las prácticas culturales propias del escenario transfronterizo.

De la frontera entre México y Estados Unidos, la región Tijuana-San Diego es el espacio binacional más importante, principalmente por el dinamismo e interacción socioeconómica observada, ya que se destaca por un elevado crecimiento urbano y la interacción de flujos de capital, trabajadores y mercancías, que impulsan la expansión económica y demográfica de la misma región (Alegría, 2010). Pero también por compartir grupos de estudiantes que transitan entre dos sistemas educativos con profundas desigualdades en la calidad de la enseñanza, infraestructura y aprendizajes. Situaciones que representan importantes retos en lo que a planeación transfronteriza se refiere (Guillén, 1993).

La estabilidad de las relaciones bilaterales entre las ciudades fronterizas de ambos países ha llevado a los gobiernos locales y estatales (Baja California en México y California en Estados Unidos) hacia la definición e instrumentación de agendas públicas transfronterizas en temas de interés común: delincuencia y seguridad ciudadana, medio ambiente, planificación municipal y regional, desarrollo económico, migración y derechos humanos, transferencia de tecnología, turismo médico, etcétera.

Sin embargo, los problemas sobre migración de estudiantes y calidad de los sistemas educativos son invisibles e irrelevantes para la agenda binacional, a pesar de que más de siete mil niños y adolescentes que viven en Tijuana cruzan a diario para estudiar en las escuelas de San Diego (Rocha y Orraca, 2018), y que en promedio ocho de cada cien alumnos de educación básica en Baja California son considerados como estudiantes transnacionales por tener trayectorias escolares fuera del sistema educativo nacional (López, 2015).

En ese sentido, para que los ciudadanos mexicanos y estadounidenses migrantes tengan garantizado su derecho a una educación de calidad, como se señala en la Agenda para el Desarrollo Sostenible (CEPAL, 2016), el primer paso consiste en hacer frente a los problemas que condicionan la inclusión, equi- 
Revista UniversidAd en DiÁlogo • Vol. 9, N. ${ }^{\circ}$ 1, Enero-Junio, 2019 • 63-85

ISSN 2215-2849 • EISSN: 2215-4752

URL: http://www.revistas.una.ac.cr/index.php/dialogo/index CORREO ELECTRÓNICO: universidadendialogo@una.cr

DOI: https://doi.org/10.15359/udre.9-1.4

dad y calidad de los procesos formativos y la promoción de oportunidades de aprendizaje para toda la vida. De ahí que los sistemas educativos deben garantizar que la enseñanza que reciben niños y jóvenes sea de calidad y produzca aprendizajes significativos demostrables.

Relacionado con este aspecto, se tiene que los estudiantes transnacionales que asisten a escuelas mexicanas se encuentran, sin generalizar, con insuficiencia de apoyo académico, desmotivados y en un escenario social-educativo muy diferente, al que no se adaptan con facilidad. Como resultado, son considerados como "alumnos en riesgo" con una fuerte tendencia a abandonar la escuela (deserción escolar) o a repetir algún grado (reprobación escolar) (Zúñiga, 2008), por lo que las implicaciones para la calidad de los sistemas educativos son más que aspectos superficiales, impertinentes y banales.

En este sentido, el objetivo de este documento es describir el panorama actual de la calidad de los aprendizajes en los sistemas escolares de California y Baja California, mediante la comparación de los resultados alcanzados en evaluaciones internacionales a gran escala, para identificar las implicaciones que tiene este horizonte educativo en la adaptación de los estudiantes transnacionales.

\section{Calidad de los sistemas educativos de California y Baja California}

En las últimas décadas, la calidad de la enseñanza y el aprendizaje se convirtió en un fenómeno de estudio común entre los investigadores de la educación. El surgimiento como unidad de análisis para indagar sobre el rendimiento de los estudiantes, de los profesores, de la escuela y del sistema educativo no es reciente. El origen de la evaluación en el contexto pedagógico se remonta al siglo XIX en Estados Unidos con la aplicación de las primeras pruebas de rendimiento estudiantil, consideradas como fuente básica de información para evaluar a las escuelas y al profesorado (Alcaraz, 2015).

Aunque para Escudero (2003) es a partir de 1930 cuando la evaluación educativa se establece como un área de conocimiento, gracias a la aportación de Ralph W. Tyler, que señalaba la necesidad de una evaluación científica que sirviera para perfeccionar la calidad de la educación, y quien años más tarde reforzaría su planteamiento al introducir el primer método sistemático de evaluación educativa.

Recientemente, el desarrollo de la misma disciplina es tan amplio que la academia no es la única interesada en el análisis de los sujetos, procesos y resultados educativos. Los medios de comunicación masiva ingresaron a la discusión pedagógica por la introducción del término educación de calidad a los 
URL: http://www.revistas.una.ac.cr/index.php/dialogo/index

CORREO ELECTRÓNICO: universidadendialogo@una.cr

DOI: https://doi.org/10.15359/udre.9-1.4

discursos y documentos oficiales, y la difusión mundial de los resultados sobre el rendimiento estudiantil en los principales estudios internacionales: TIMSS, ${ }^{3}$ PIRLS, ${ }^{4}$ PISA $^{5}$ y PERCE. ${ }^{6}$ En otras palabras, las dos situaciones atrajeron la atención de propios y extraños para opinar sobre la calidad de la enseñanza y del aprendizaje en los sistemas escolares participantes.

Una crítica al modelo evaluativo vigente consiste en que los sistemas educativos se paralizan cuando se inicia la aplicación de una prueba estandarizada, ya que todo lo que se hace con anterioridad a la misma se convierte en una preparación para superarla (Casanova, 2012). Adicionalmente, "las nuevas pretensiones de evaluar en forma masiva la calidad no es un asunto estrictamente académico, es también político pues se produce un cambio en la institución escolar, en el sistema educativo y en los agentes que lo habitan” (Martínez, 2008, p. 22).

El primer estudio responsable de evaluar un sistema educativo completo estuvo dirigido por James Coleman en Estados Unidos en la década de 1960. El Informe Coleman (1966), como popularmente es conocido, examinó la relación entre los diversos factores (escolares e individuales) y los logros educativos, utilizando una muestra significativa de seiscientos mil estudiantes, setenta mil docentes y cuatro mil escuelas.

Sin embargo, es hasta la década de 1990 cuando la Asociación Internacional para la Evaluación del Logro Educativo (IEA, por sus siglas en inglés), la Oficina Regional de Educación para América Latina y el Caribe (OREALC) y la Organización para la Cooperación y el Desarrollo Económicos (OCDE) iniciaron la creación de las primeras evaluaciones internacionales con el objetivo de medir y comparar la calidad de los sistemas educativos.

Las dos evaluaciones administradas por IEA son TIMSS y PIRLS, aplicadas por primera vez en 1995 y 2001, respectivamente; la OREALC implementó su propia estrategia evaluativa regional en 1997 bajo el nombre de PERCE; y la OCDE instrumentó con la llegada del año 2000 la evaluación internacional más popular, la prueba PISA (Díaz y Osuna, 2016). Hoy en día estas evaluaciones a gran escala son un referente obligado cuando se debate pedagógicamente sobre calidad de la educación.

3 Estudio Internacional de Tendencias en Matemáticas y Ciencias

$4 \quad$ Estudio Internacional de Progreso en Comprensión Lectora

5 Programa para la Evaluación Internacional de Estudiantes

6 Primer Estudio Regional Comparativo y Explicativo para América Latina y el Caribe 

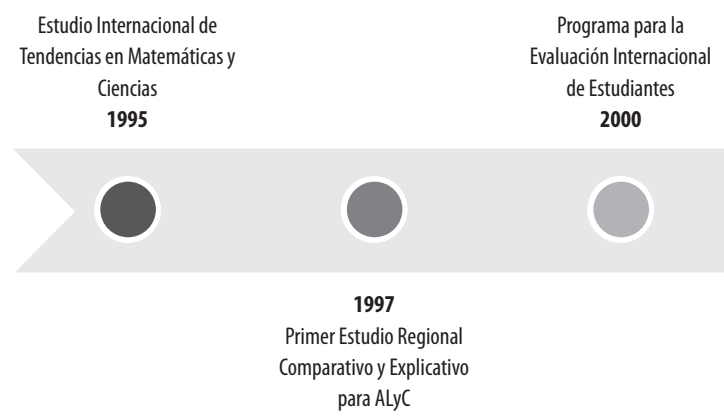

Figura 1. Orden histórico de la primera aplicación de las cuatro evaluaciones internacionales del aprendizaje.

Fuente: Elaboración propia con información de Díaz y Osuna, 2016.

Desde la primera aplicación, Estados Unidos participa activamente en tres evaluaciones internacionales: TIMSS, PIRLS y PISA; mientras que México solo evalúa a sus estudiantes a través de dos instrumentos: PISA y la prueba regional de la OREALC, en TIMSS únicamente fue partícipe en la edición de 1995 (ver tabla 1).

Tabla 1

Participación de Estados Unidos y México en las evaluaciones internacionales y regionales más populares

\begin{tabular}{lll}
\hline Evaluación/Prueba & \multicolumn{1}{c}{ Estados Unidos } & México \\
\hline TIMSS & $1995, \quad 1999,2003,2007$, & 1995 \\
& 2011,2015 & \\
\hline LLECE $^{*}$ & Sin participación & $1997,2006,2013$ \\
\hline PISA & $2000,2003,2006,2009$, & $2000,2003,2006,2009,2012$, \\
& 2012,2015 & 2015 \\
\hline PIRLS & $2001,2006,2011,2016$ & Sin participación \\
\hline
\end{tabular}

* Laboratorio Latinoamericano de Evaluación de la Calidad de la Educación (LLECE) de la OREALC.

Fuente: elaboración propia.

Tomando como referencia la prueba PISA, una estrategia evaluativa común entre los sistemas educativos de Estados Unidos y México, se observa una notable desigualdad en el logro de los aprendizajes clave de ambos países, así como entre los estados subnacionales que los integran. 
URL: http://www.revistas.una.ac.cr/index.php/dialogo/index

CORREO ELECTRÓNICO: universidadendialogo@una.cr

DOI: https://doi.org/10.15359/udre.9-1.4

Ejemplo de lo anterior queda evidenciado en el último reporte de PISA 2015, donde se muestra que, de los setenta países y regiones evaluadas, Estados Unidos y México ocuparon la posición 25 y 58 respectivamente; mientras que entre el grupo de países miembros de la OCDE Estados Unidos se posicionó en el lugar 19 y México (35) se mantuvo como el país con el peor resultado en Ciencias, Lectura y Matemáticas (OCDE, 2016) (ver tabla 2).

Tabla 2

Los sistemas educativos con mejores resultados en PISA 2015

\begin{tabular}{clcclc}
\hline \multicolumn{2}{c}{ Países y regiones participantes } & \multicolumn{2}{c}{ Países miembros de la OCDE } \\
\hline Posición & \multicolumn{1}{c}{ País } & $\begin{array}{c}\text { Rendimiento en } \\
\text { Ciencias }\end{array}$ & Posición & País & $\begin{array}{c}\text { Rendimiento } \\
\text { en Ciencias }\end{array}$ \\
\hline 1 & Singapur & 556 & 1 & Japón & 538 \\
\hline 2 & Japón & 538 & 2 & Estonia & 534 \\
\hline 3 & Estonia & 534 & 3 & Finlandia & 531 \\
\hline 4 & China Taipéi & 532 & 4 & Canadá & 528 \\
\hline 5 & Finlandia & 531 & 5 & Corea del Sur & 516 \\
\hline 6 & Macao & 529 & 6 & Nueva Zelanda & 513 \\
\hline 7 & Canadá & 528 & 7 & Eslovenia & 513 \\
\hline 8 & Vietnam & 525 & 8 & Australia & 510 \\
\hline 9 & Hong Kong & 523 & 9 & Reino Unido & 509 \\
\hline 10 & P-S-G-J* & 518 & 10 & Alemania & 509 \\
\hline 25 & Estados Unidos & 496 & 19 & Estados Unidos & 496 \\
\hline 58 & México & 416 & 35 & México & 416 \\
\hline & Media OCDE & 493 & & Media OCDE & 493 \\
\hline
\end{tabular}

* Pekín, Shanghai, Jiangsu y Guangdong, en China.

Fuente: Elaboración propia con datos de la OCDE, 2016.

Al realizar un contraste de los resultados educativos y enfatizando la diferencia en el puntaje alcanzado en matemáticas, lectura y ciencias entre los estudiantes de ambos países, llama la atención la gran diferencia entre el rendimiento de los estudiantes de Estados Unidos y los de México. Los jóvenes estadounidenses alcanzaron un rendimiento medio en PISA 2015 de 496 puntos en 
Revista UniversidAd en DiÁlogo • Vol. 9, N. ${ }^{\circ}$ 1, Enero-Junio, 2019 • 63-85

ISSN 2215-2849 • EISSN: 2215-4752

URL: http://www.revistas.una.ac.cr/index.php/dialogo/index CORREO ELECTRÓNICO: universidadendialogo@una.cr

DOI: https://doi.org/10.15359/udre.9-1.4

Ciencias, 497 en Lectura y 470 en Matemáticas; por el contrario, los estudiantes mexicanos consiguieron puntajes de 416, 423 y 408 respectivamente; sin embargo, en ambos países se observa una disminución en el rendimiento de los estudiantes en los tres dominios, en el periodo 2009-2015 (ver tabla 3).

Por lo tanto, se infiere que al finalizar la educación obligatoria el rendimiento estudiantil en Estados Unidos es muy superior al de México, pero inferior al de Japón, Estonia y Finlandia, países de la OCDE que lideran los tres dominios.

Tabla 3

Resultados educativos de Estados Unidos y México en PISA 2009, 2012 y 2015

\begin{tabular}{ccccc}
\hline Dominio & Año & Estados Unidos & México & Media OCDE \\
\hline \multirow{4}{*}{ Matemáticas } & 2009 & 487 & 419 & 496 \\
\cline { 2 - 5 } & 2012 & 481 & 413 & 494 \\
\cline { 2 - 5 } & 2015 & 470 & 408 & 490 \\
\hline \multirow{4}{*}{ Lectura } & 2009 & 500 & 425 & 493 \\
\cline { 2 - 5 } & 2012 & 498 & 424 & 496 \\
\hline \multirow{3}{*}{ Ciencias } & 2015 & 497 & 423 & 493 \\
& 2009 & 502 & 416 & 501 \\
\cline { 2 - 5 } & 2012 & 497 & 415 & 501 \\
\hline
\end{tabular}

Fuente: Elaboración propia con datos de la OCDE, 2016.

Realizando una comparación entre las entidades federativas mexicanas, se tiene que en el año 2012 Baja California se ubicó dentro de los quince estados con desempeño aceptable, los resultados en Matemáticas, Lectura y Ciencias fueron 415, 428 y 417 respectivamente, un par de puntos por encima del promedio nacional; en Matemáticas los líderes son Aguascalientes (437), Nuevo León (436), Jalisco (435), Querétaro (434) y Colima (429) (INEE, 2013).

En Ciencias, de nueva cuenta, Jalisco (436), Nuevo León (435), Aguascalientes (435), Querétaro (432) y Colima (429) ocuparon las primeras posiciones; los estados más rezagados son Guerrero (372), Chiapas (377) y Tabasco (391), que casualmente son entidades federativas del sureste mexicano, con altos índices de pobreza (INEE, 2013). 
URL: http://www.revistas.una.ac.cr/index.php/dialogo/index

CORREO ELECTRÓNICO: universidadendialogo@una.cr

DOI: https://doi.org/10.15359/udre.9-1.4

Por otra parte, tomando en consideración los estados de USA, se tiene que en California no se aplicó el examen PISA 2012 y 2015, ya que la muestra estuvo constituida por estudiantes de las entidades de Massachusetts (2015 y 2012), North Carolina (2015), Connecticut (2012) y Florida (2012). No obstante, si se analiza el informe de TIMSS 2011, el puntaje alcanzado por los alumnos californianos de octavo grado en Matemáticas (493) y Ciencias (499) se ubica por debajo del promedio nacional (509 en Matemáticas y 525 en Ciencias) e internacional (500 en los dos dominios) (NCES, 2013).

Al comparar al mismo sistema educativo con el resto de las entidades norteamericanas evaluadas, vemos que en Matemáticas y Ciencias solo supera a Alabama (466 y 485, respectivamente), el estado con más debilidades académicas, y tiene una posición inferior con respecto a los sistemas de Massachusetts (561 y 567), Minnesota (545 y 553), North Carolina (537 y 532), Indiana (522 y 533), Connecticut (518 y 532), Colorado (518 y 542) y Florida (513 y 530) (NCES, 2013) (ver tabla 4).

Tabla 4

Rendimiento de los estudiantes estadounidenses en TIMSS 2011

\begin{tabular}{lcc}
\hline Entidad federativa & Rendimiento en Matemáticas & Rendimiento en Ciencias \\
\hline Massachusetts & 561 & 567 \\
\hline Minnesota & 545 & 553 \\
\hline North Carolina & 537 & 532 \\
\hline Indiana & 522 & 533 \\
\hline Connecticut & 518 & 532 \\
\hline Colorado & 518 & 542 \\
\hline Florida & 513 & 530 \\
\hline California & 493 & 499 \\
\hline Alabama & 466 & 485 \\
\hline
\end{tabular}

Fuente: Elaboración propia con datos del NCES, 2013.

En síntesis, a pesar de que California y Baja California son estados fronterizos con una integración económica y cultural consolidada, seguimos observando una profunda dispersión en asuntos educativos. Por ejemplo, no existe ningún instrumento de evaluación común por medio del cual se puedan establecer comparaciones objetivas y congruentes sobre la calidad de los dos sistemas escolares, ni existe una agenda pública de educación para afrontar aquellos 
Revista UniversidAd en DiÁlogo • Vol. 9, N. ${ }^{\circ}$ 1, Enero-Junio, 2019 • 63-85

ISSN 2215-2849 • EISSN: 2215-4752

URL: http://www.revistas.una.ac.cr/index.php/dialogo/index CORREO ELECTRÓNICO: universidadendialogo@una.cr

DOI: https://doi.org/10.15359/udre.9-1.4

problemas que trascienden fronteras, ni mucho menos programas regionales para mejorar los procesos de enseñanza y rendimiento de los estudiantes en las evaluaciones internacionales del aprendizaje.

Un aspecto común es que ambos tienen comunidades estudiantiles con debilidades en la demostración de competencias o aprendizajes universales (ver tabla 5); principalmente, las que se relacionan con perspectivas de lectura y cognición, conocimientos básicos de aritmética y matemáticas, así como ciencia y tecnología (UNESCO, 2013).

Tabla 5

Dominios del aprendizaje universal

\begin{tabular}{|c|c|c|}
\hline Dominio & Descripción & Ejemplos de subdominio \\
\hline $\begin{array}{l}\text { Bienestar } \\
\text { físico }\end{array}$ & $\begin{array}{l}\text { Cómo los niños y los jóvenes utilizan } \\
\text { sus cuerpos, desarrollan control motor y } \\
\text { comprenden y exhiben nutrición adecuada, } \\
\text { ejercicio, higiene y prácticas de seguridad. }\end{array}$ & $\begin{array}{l}\text { - Salud física e higiene } \\
\text { - Alimentación y nutrición } \\
\text { - Actividad física }\end{array}$ \\
\hline $\begin{array}{l}\text { Social y } \\
\text { emocional }\end{array}$ & $\begin{array}{l}\text { Cómo los niños y los jóvenes fomentan y } \\
\text { mantienen relaciones con adultos y coetáneos. } \\
\text { También, cómo se perciben a sí mismos en } \\
\text { relación con otros. }\end{array}$ & $\begin{array}{l}\text { - Valores de la comunidad y } \\
\text { sociales } \\
\text { - Valores cívicos } \\
\text { - Salud mental y bienestar }\end{array}$ \\
\hline $\begin{array}{l}\text { Cultura y las } \\
\text { artes }\end{array}$ & $\begin{array}{l}\text { Expresión creativa, incluyendo actividades } \\
\text { de las áreas de música, teatro, danza o } \\
\text { movimiento creativo y de las artes visuales, } \\
\text { medios y literarias. También las experiencias } \\
\text { culturales en familia, en la escuela, la } \\
\text { comunidad y el país. }\end{array}$ & $\begin{array}{l}\text { - Artes creativas } \\
\text { - Conocimiento cultural } \\
\text { - Identidad propia y de la } \\
\text { comunidad } \\
\text { - Conciencia de y respeto } \\
\text { por la diversidad }\end{array}$ \\
\hline $\begin{array}{l}\text { Alfabetismo y } \\
\text { comunicación }\end{array}$ & $\begin{array}{l}\text { Comunicación en el primer idioma o los } \\
\text { primeros idiomas de la sociedad en que los } \\
\text { niños y jóvenes viven, incluyendo hablar, } \\
\text { escuchar, leer, escribir y comprender la } \\
\text { palabra hablada y escrita en diversos medios. }\end{array}$ & $\begin{array}{l}\text { - Hablar y escuchar } \\
\text { - Vocabulario } \\
\text { - Escritura } \\
\text { - Lectura }\end{array}$ \\
\hline $\begin{array}{l}\text { Enfoques de } \\
\text { aprendizaje y } \\
\text { cognición }\end{array}$ & $\begin{array}{l}\text { Los enfoques de aprendizaje describen el } \\
\text { compromiso del aprendiz, la motivación y la } \\
\text { participación en el aprendizaje. La cognición es } \\
\text { el proceso mental para adquirir el aprendizaje } \\
\text { por medio de estos enfoques diferentes. }\end{array}$ & $\begin{array}{l}\text { - Perseverancia y atención } \\
\text { - Cooperación } \\
\text { - Solución de problemas } \\
\text { - Autodirección } \\
\text { - Pensamiento crítico }\end{array}$ \\
\hline
\end{tabular}


URL: http://www.revistas.una.ac.cr/index.php/dialogo/index CORREO ELECTRÓNICO: universidadendialogo@una.cr DOI: https://doi.org/10.15359/udre.9-1.4

\begin{tabular}{|c|c|c|}
\hline $\begin{array}{l}\text { Aritmética y } \\
\text { matemáticas }\end{array}$ & $\begin{array}{l}\text { La ciencia de números y el lenguaje } \\
\text { cuantitativo utilizado universalmente para } \\
\text { representar fenómenos observados } \\
\text { en el entorno. }\end{array}$ & $\begin{array}{l}\text { - Numerar conceptos y } \\
\text { operaciones } \\
\text { - Geometría y patrones } \\
\text { - Aplicación de } \\
\text { matemáticas } \\
\text { - Datos y estadísticas }\end{array}$ \\
\hline $\begin{array}{l}\text { Ciencia y } \\
\text { tecnología }\end{array}$ & $\begin{array}{l}\text { La ciencia es el conocimiento específico, } \\
\text { entidad o sistema de conocimiento que } \\
\text { cubre leyes físicas y verdades generales. La } \\
\text { tecnología hace referencia a la creación y al } \\
\text { uso de herramientas para resolver problemas. }\end{array}$ & $\begin{array}{l}\text { - Información científica } \\
\text { - Ciencias de la vida } \\
\text { - Ciencias físicas } \\
\text { - Ciencias de la tierra } \\
\text { - Conciencia y uso de la } \\
\text { tecnología digital }\end{array}$ \\
\hline
\end{tabular}

Fuente: UNESCO, 2013.

\section{Estudiantes trasnacionales en la región Tijuana-San Diego}

Las instituciones educativas de México y Estados Unidos no están exentas de la presencia de minorías estudiantiles con rasgos étnicos, lingüísticos, culturales y religiosos específicos, pero también con estatus legal y trayectorias académicas diferentes, que los distinguen del resto de la matrícula escolar. Por ejemplo, hay estudiantes asiáticos, hispanos, negros, centroamericanos, haitianos, estadounidenses de origen mexicano, indígenas, ilegales, migrantes internos, con doble ciudadanía, etcétera; pero el común denominador de los ejemplos anteriores es que son estudiantes migrantes internacionales, o bien, pueden llegar a serlo.

Zúñiga propone una tipología para clasificar a la misma comunidad estudiantil en dos grupos básicos: transnacionales y binacionales; los primeros son aquellos alumnos que transitan de un sistema escolar a otro, y los segundos son los que poseen la doble nacionalidad. Sin embargo, debido a que los dos grupos no son excluyentes, "es posible encontrar estudiantes transnacionales que son binacionales o alumnos binacionales que no son considerados transnacionales y, por supuesto, transnacionales que no son binacionales" (2013, p. 4).

Adicionalmente, la Secretaría de Educación Pública (SEP) solo reconoce a los estudiantes transnacionales, y se refiere a ellos como "migrantes de retorno que tienen trayectorias escolares fragmentadas, es decir, que iniciaron sus estudios en un país para luego continuar en otro" (Jalili, 2015, p. 11). 
Revista Universidad en DiÁlogo • Vol. 9, N. ${ }^{\circ}$ 1, Enero-Junio, 2019 • 63-85

ISSN 2215-2849 • EISSN: 2215-4752

URL: http://www.revistas.una.ac.cr/index.php/dialogo/index CORREO ELECTRÓNICO: universidadendialogo@una.cr

DOI: https://doi.org/10.15359/udre.9-1.4

En contraposición, el $\mathrm{PROBEM}^{7}$ en Estados Unidos admite únicamente la categoría de estudiante migrante binacional, que es aquel alumno que se traslada de México a EUA con sus padres, o como un joven emancipado al menos una vez en los últimos 36 meses (Elorriaga, Blancas y Ramos, 2015).

Tabla 6

Tipos de estudiantes migrantes en las escuelas de la región Tijuana-San Diego

\begin{tabular}{lll}
\hline Estudiantes nacionales & Estudiantes binacionales & Estudiantes transnacionales \\
\hline Son aquellos alumnos & Son los alumnos que tienen & Son aquellos alumnos que \\
originarios de cada país, & legalmente una doble & sin importar su origen \\
con una trayectoria escolar & nacionalidad, pero que & o nacionalidad tienen \\
dentro del mismo sistema & no necesariamente tienen & trayectorias escolares en dos \\
educativo nacional. & $\begin{array}{l}\text { experiencias académicas en dos } \\
\text { o más sistemas educativos. }\end{array}$ & o más sistemas educativos. \\
\hline
\end{tabular}

Fuente: Elaboración propia con información de Osuna, Miramontes y Villaseñor, 2018.

Por último, Osuna, Miramontes y Villaseñor (2018), a partir de la clasificación de Zúñiga (2013), reconceptualizan los grupos de estudiantes y agregan una categoría adicional para que la misma sea aplicable a los migrantes internos y externos. Como resultado, se reconocen tres tipos de minorías estudiantiles (nacionales, binacionales y transnacionales) (ver tabla 6):

Los estudiantes nacionales son aquellos originarios de cada país, con una trayectoria escolar dentro del mismo sistema educativo nacional, transitan de un sistema estatal a otro, pero administrativamente dependen del sistema federal. Los binacionales son estudiantes que tienen legalmente una doble nacionalidad (mexicana-estadounidense, mexicana-colombiana, mexicana-cubana, etcétera) pero que no necesariamente tienen experiencias académicas en dos o más sistemas educativos. Por último, los estudiantes transnacionales son aquellos que sin importar su origen o nacionalidad tienen trayectorias escolares en dos o más sistemas educativos. (Osuna, Miramontes y Villaseñor, 2018, p. 6)

Tomando como referencia la caracterización anterior, se analiza la situación de los estudiantes transnacionales en las escuelas de la región Tijuana-San Diego.

\footnotetext{
Programa Binacional de Educación Migrante. Tiene entre sus objetivos nacionales: realizar acciones que aseguren la continuidad escolar de los niños, jóvenes y adultos migrantes de ambos países; contribuir para que se ofrezca a los menores de edad que migran entre México y Estados Unidos de América una educación de calidad, equidad y pertinencia; así como fortalecer las relaciones educativas y socioculturales entre los dos países a través del intercambio de experiencias culturales y académicas con las autoridades educativas y docentes (PROBEM México, 2015).
} 
URL: http://www.revistas.una.ac.cr/index.php/dialogo/index

CORREO ELECTRÓNICO: universidadendialogo@una.cr

DOI: https://doi.org/10.15359/udre.9-1.4

\section{Estudiantes transnacionales en Baja California, México}

Baja California es junto con Jalisco, Michoacán, Estado de México y Guanajuato las entidades federativas mexicanas que más connacionales repatriados reciben anualmente; pero si hablamos de los niños y jóvenes en la misma condición migratoria y en edad suficiente para continuar su educación obligatoria, los estados líderes son Oaxaca, Guerrero, Sonora, Tamaulipas y Michoacán, que durante el año 2015 recibieron en promedio a 5645 menores deportados (Lorenzen, 2016; Serrano y Jaramillo, 2017).

\section{Tabla 7}

Caracterización de los estudiantes transnacionales en escuelas de México, según el origen

\begin{tabular}{lrc}
\multicolumn{1}{c}{ Origen } & Cantidad & Porcentaje \\
\hline Estados Unidos & 355,983 & $99.4 \%$ \\
\hline Otro país & 2,162 & $0.6 \%$ \\
\hline Total & 358,145 & $100 \%$ \\
\hline
\end{tabular}

Fuente: Elaboración propia con datos de Jalili Zúñiga, 2015.

De acuerdo con estadísticas oficiales de la Dirección General de Relaciones Internacionales de la SEP, en diez años (2000-2010) el retorno de migrantes mexicanos se incrementó en un cien por ciento al pasar de 667 mil a 1.4 millones de connacionales repatriados; donde el 25\% eran menores de 19 años, aproximadamente 355 mil niños, niñas y adolescentes procedentes de Estados Unidos y a los que el Gobierno de México les debe garantizar el derecho a recibir una educación de calidad (Jalili Zúñiga, 2015).

Tabla 8

Caracterización de los estudiantes transnacionales en escuelas de México, según el género

\begin{tabular}{llc}
\hline Género & Cantidad & Porcentaje \\
\hline Hombre & 178,714 & $49.9 \%$ \\
\hline Mujer & 179,431 & $50.1 \%$ \\
\hline Total & 358,145 & $100 \%$ \\
\hline
\end{tabular}

Fuente: Elaboración propia con datos de Jalili Zúñiga, 2015. 
Revista UniversidAd en DiÁlogo • Vol. 9, N. ${ }^{\circ}$ 1, Enero-Junio, 2019 • 63-85

ISSN 2215-2849 • EISSN: 2215-4752

URL: http://www.revistas.una.ac.cr/index.php/dialogo/index CORREO ELECTRÓNICO: universidadendialogo@una.cr

DOI: https://doi.org/10.15359/udre.9-1.4

En el año 2013, de la totalidad de niños y jóvenes repatriados al territorio nacional, solo el $96.8 \%$ asistieron a la escuela para continuar su formación académica, por lo que existe una minoría de 11840 menores a los que por motivos desconocidos se les negó el derecho de aprender. De los que sí asisten, el grueso de la matrícula transnacional se localiza en la enseñanza primaria (57\%), posteriormente en preescolar (19.2\%), secundaria (17.2\%), y en menor proporción, en la educación media superior u otro (6.6\%); además, los principales estados que más reciben en sus centros educativos a este tipo de estudiantes son: Chihuahua (11.9\%), Baja California (11.8\%), Jalisco (9.2\%), Sonora (6.4\%) y Estado de México (6.1\%) (Jalili, 2015).

En particular, el sistema educativo bajacaliforniano, con apoyo del PROBEM estatal, llevó a cabo durante el ciclo escolar 2013-14 la inscripción de 52,879 alumnos procedentes de 28 países, ${ }^{8}$ de los cuales el $57.2 \%$ fueron atendidos en el municipio de Tijuana, 25.2\% en Mexicali, 9.5\% en Ensenada, 4.6\% en Playas de Rosarito y 3.5\% en Tecate (Sistema Educativo Estatal, 2015) (ver figura 2).

Adicionalmente, por nivel educativo, el $62.2 \%$ de los estudiantes transnacionales se incorporó a la enseñanza primaria, el $19.2 \%$ a preescolar y el $18.7 \%$ a secundaria (López, 2015).

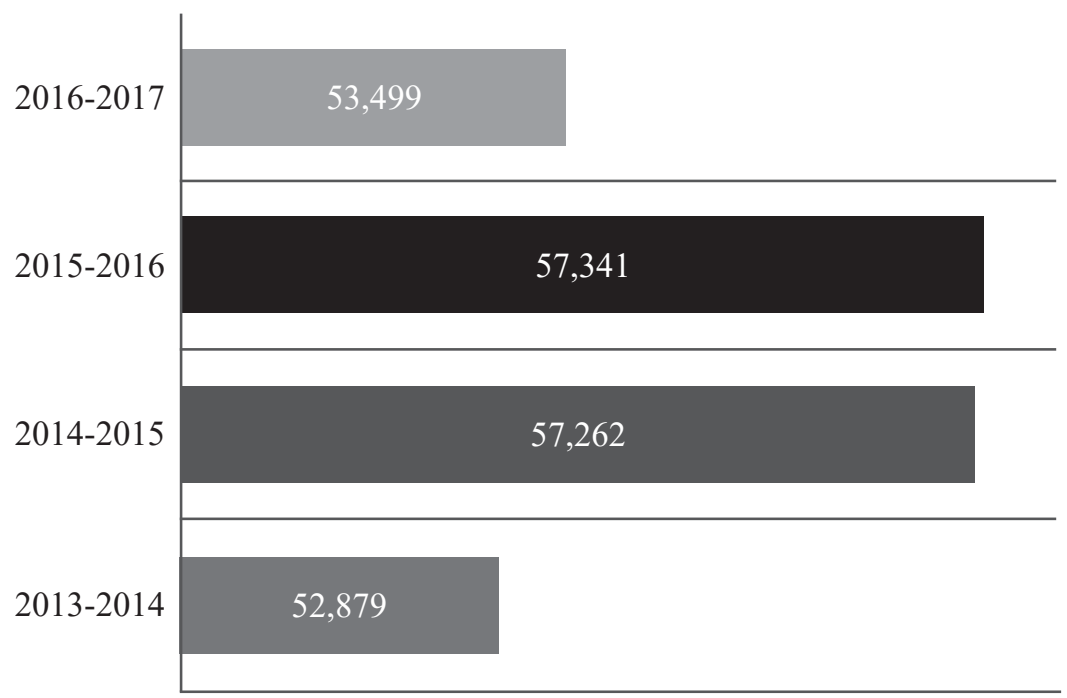

Figura 2. Matrícula de estudiantes transnacionales en Baja California por ciclo escolar. Fuente: Elaboración propia con datos del Sistema Educativo Estatal.

E1 90\% de los estudiantes transnacionales se trasladó de Estados Unidos a Baja California, en México 
URL: http://www.revistas.una.ac.cr/index.php/dialogo/index

CORREO ELECTRÓNICO: universidadendialogo@una.cr

DOI: https://doi.org/10.15359/udre.9-1.4

Posteriormente, en el año escolar 2014-15 hubo un incremento en la matrícula transnacional de la educación básica al pasar de 52879 a 57262 estudiantes (Gobierno de Baja California, 2015), aunque, según las estadísticas del mismo PROBEM, únicamente se formalizó la inscripción de 54 109, donde la mayor cantidad de niños y jóvenes se mantuvo en primaria (62.3\%), después en secundaria (20.6\%) y, finalmente, en preescolar (17.1\%); además, debemos destacar el caso de las escuelas del municipio de Tijuana, que en los ciclos 2013-14 y 2014-15 hicieron grandes esfuerzos para acoger en sus aulas a más del 55\% de la totalidad de alumnos migrantes internacionales por nivel educativo (López, 2015) (ver tabla 10).

Tabla 10

Distribución de los estudiantes transnacionales por municipio y ciclo escolar

\begin{tabular}{lrrrrrr}
\hline \multirow{2}{*}{ Municipios } & \multicolumn{3}{c}{ Ciclo escolar 2013-2014 } & \multicolumn{3}{c}{ Ciclo escolar 2014-2015 } \\
\cline { 2 - 7 } & Preescolar & Primaria & Secundaria & Preescolar & Primaria & Secundaria \\
\hline Ensenada & 884 & 3,068 & 1,039 & 758 & 3,109 & 1,145 \\
\hline Mexicali & 2,711 & 8,292 & 2,332 & 2,486 & 8,158 & 2,659 \\
\hline Tijuana & 5,841 & 18,695 & 5,692 & 5,267 & 19,481 & 6,482 \\
\hline Tecate & 358 & 1,172 & 372 & 365 & 1,222 & 399 \\
\hline Playas de Rosarito & 391 & 1603 & 429 & 379 & 1,727 & 472 \\
\hline Total & 10,185 & 32,892 & 9,864 & 9,255 & 33,697 & 11,157 \\
\hline
\end{tabular}

Fuente: López, 2015.

Para finalizar, en el ciclo escolar 2015-16 la cifra de estudiantes transnacionales a nivel estatal se incrementó a 57 341, para luego presentar una reducción significativa en más de 3800 niños, niñas y adolescentes durante el periodo 2016-17 (Gobierno de Baja California, 2016 y 2017).

El comportamiento de este panorama educativo-migratorio en Baja California puede estar asociado al hecho de que los connacionales repatriados a México prefieren radicar con sus familias en ciudades adyacentes a la frontera con Estados Unidos, debido a que sus estados o localidades de origen no ofrecen las oportunidades laborales y escolares esperadas para ellos y sus progenitores. Lo anterior tiene efectos en el sistema educativo estatal porque, en la mayoría de los casos, los mexicanos deportados vienen acompaños de sus hijos con la esperanza de continuar sus estudios en escuelas públicas de la región, por 
Revista UniversidAd en DiÁlogo • Vol. 9, N. ${ }^{\circ}$ 1, Enero-Junio, 2019 • 63-85

ISSN 2215-2849 • EISSN: 2215-4752

URL: http://www.revistas.una.ac.cr/index.php/dialogo/index CORREO ELECTRÓNICO: universidadendialogo@una.cr

DOI: https://doi.org/10.15359/udre.9-1.4

lo que al formalizar su ingreso automáticamente se convierten en estudiantes transnacionales. O bien, viven del lado mexicano, pero se forman en las escuelas estadounidenses cercanas a la línea fronteriza, lo que tiene implicaciones para los dos sistemas de enseñanza de la región Tijuana-San Diego.

\section{Estudiantes transnacionales en California, Estados Unidos}

El estado de California se caracteriza por contar con la tasa de población mexicana más alta de Estados Unidos. En 2015, residían en la entidad 4370094 migrantes mexicanos, es decir, aproximadamente 36 de cada 100 connacionales que viven en el vecino país del norte; el resto se divide entre los estados de Texas, Illinois, Arizona, Florida, Georgia, Carolina del Norte y otros (Serrano y Jaramillo, 2017).

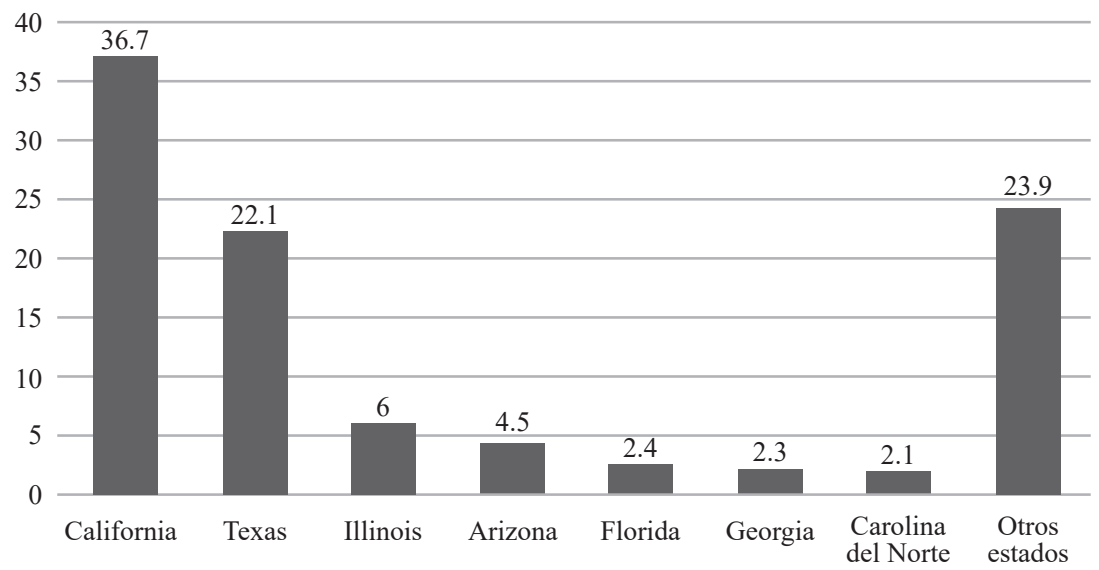

Figura 3. Porcentaje de migrantes mexicanos en Estados Unidos por entidad, 2015.

Fuente: Elaboración propia con datos de Serrano y Jaramillo, 2017.

Además de ser un estado líder en población y recepción de migrantes internacionales, California tiene el sistema educativo más grande de la Unión Americana, con 10462 escuelas, 6226737 estudiantes y 263474 profesores (ver cuadro 11); seguido por los sistemas de Texas, Nueva York, Florida e Illinois (NCES, 2016).

Durante el ciclo escolar 2016-17 el sistema educativo californiano registró una población estudiantil de 6228235 niños y jóvenes en los 1024 distritos escolares; según la distribución étnica de la matrícula (ver cuadro 12), el 54.24\% de los alumnos son hispanos o latinos, $23.61 \%$ blancos estadounidenses y, en menor proporción, el $8.98 \%$ asiáticos, $5.62 \%$ afroamericanos, $2.47 \%$ filipinos, $0.54 \%$ 
URL: http://www.revistas.una.ac.cr/index.php/dialogo/index

CORREO ELECTRÓNICO: universidadendialogo@una.cr

DOI: https://doi.org/10.15359/udre.9-1.4

nativos americanos, y el restante $4.54 \%$ tiene otro origen étnico (California Department of Education, 2017).

Tabla 11

Características de los sistemas educativos más numerosos de Estados Unidos, 2015-16

\begin{tabular}{lccc}
\multicolumn{1}{c}{ Estado } & Escuelas & Estudiantes & Profesores \\
\hline California & 10,462 & $6,226,737$ & 263,474 \\
\hline Texas & 9,385 & $5,301,477$ & 347,328 \\
\hline Nueva York & 4,866 & $2,711,626$ & 206,086 \\
\hline Florida & 4,427 & $2,792,234$ & 182,586 \\
\hline Illinois & 4,228 & $2,041,779$ & 129,948 \\
\hline Estados Unidos* & 1,966 & 986,804 & 61,794 \\
\hline
\end{tabular}

*Promedio nacional.

Fuente: Elaboración propia con datos de NCES, 2016.

Tabla 12

Distribución étnica de los estudiantes en escuelas de California, 2016-17

\begin{tabular}{lcc}
\hline \multicolumn{1}{c}{ Ethnicity } & Number of students & Percentage \\
\hline African American not Hispanic & 350,338 & 5.62 \\
\hline American Indian or Alaska Native & 33,369 & 0.54 \\
\hline Asian & 559,159 & 8.98 \\
\hline Filipino & 153,670 & 2.47 \\
\hline Hispanic or Latino & $3,378,344$ & 54.24 \\
\hline Pacific Islander & 29,384 & 0.47 \\
\hline White not Hispanic & $1,470,499$ & 23.61 \\
\hline Two or More Races Not Hispanic & 207,170 & 3.33 \\
\hline None Reported & 46,302 & 0.74 \\
\hline Total & $6,228,235$ & 100 \\
\hline
\end{tabular}

Fuente: Departamento de Educación de California, 2017. 
Revista UniversidAd en DiÁlogo • Vol. 9, N. ${ }^{\circ}$ 1, Enero-Junio, 2019 • 63-85

ISSN 2215-2849 • EISSN: 2215-4752

URL: http://www.revistas.una.ac.cr/index.php/dialogo/index CORREO ELECTRÓNICO: universidadendialogo@una.cr

DOI: https://doi.org/10.15359/udre.9-1.4

En el condado de San Diego la situación es muy similar al escenario educativo estatal, debido a que los hispanos o latinos (48.4\%) son la población estudiantil más numerosa, le siguen los blancos estadounidenses $(30.3 \%)$, los asiáticos (6.1\%), los afroamericanos (4.7\%), los filipinos (3.9\%), los nativos americanos $(0.5 \%)$, y el resto se agrupa en la etiqueta de otro origen étnico (6.2\%) (California Department of Education, 2017).

Tabla 13

Distribución étnica de los estudiantes en los estados norteamericanos que comparten frontera con México, 2015-16

\begin{tabular}{lcccc}
\hline \multirow{2}{*}{ Ethnicity } & \multicolumn{4}{c}{ State education } \\
\cline { 2 - 5 } & Arizona & California & New Mexico & Texas \\
\hline American Indian/Alaska Native & 50,331 & 34,704 & 34,222 & 20,927 \\
\hline Asian/Pacific Islander & 34,669 & 737,831 & 4,254 & 220,840 \\
\hline Black & 58,715 & 361,752 & 6,482 & 668,829 \\
\hline Hispanic & 497,937 & $3,360,562$ & 205,853 & $2,768,431$ \\
\hline White & 437,740 & $1,500,932$ & 79,345 & $1,513,535$ \\
\hline Two or more races & 29,648 & 230,956 & 5,538 & 108,915 \\
\hline
\end{tabular}

Fuente: Elaboración propia con datos de NCES, 2016.

En ambos casos, sobresale el posicionamiento de la comunidad estudiantil hispana en el sistema educativo estadounidense, ellos ocupan más de la mitad de los espacios disponibles en las escuelas de educación obligatoria de California y San Diego, desplazando a la propia comunidad norteamericana (blancos estadounidenses) al segundo sitio.

Por último, las estadísticas del Departamento de Educación de California (2017) indican que uno de cada tres estudiantes migrantes en Estados Unidos vive en dicha entidad federativa; solo en el año escolar 2015-16 había más de 96750 niños y adolescentes transnacionales educándose en las más de diez mil escuelas estatales en ciclos regulares, así como otros 42,570 alumnos que asistían a clases de verano. 
URL: http://www.revistas.una.ac.cr/index.php/dialogo/index

CORREO ELECTRÓNICO: universidadendialogo@una.cr

DOI: https://doi.org/10.15359/udre.9-1.4

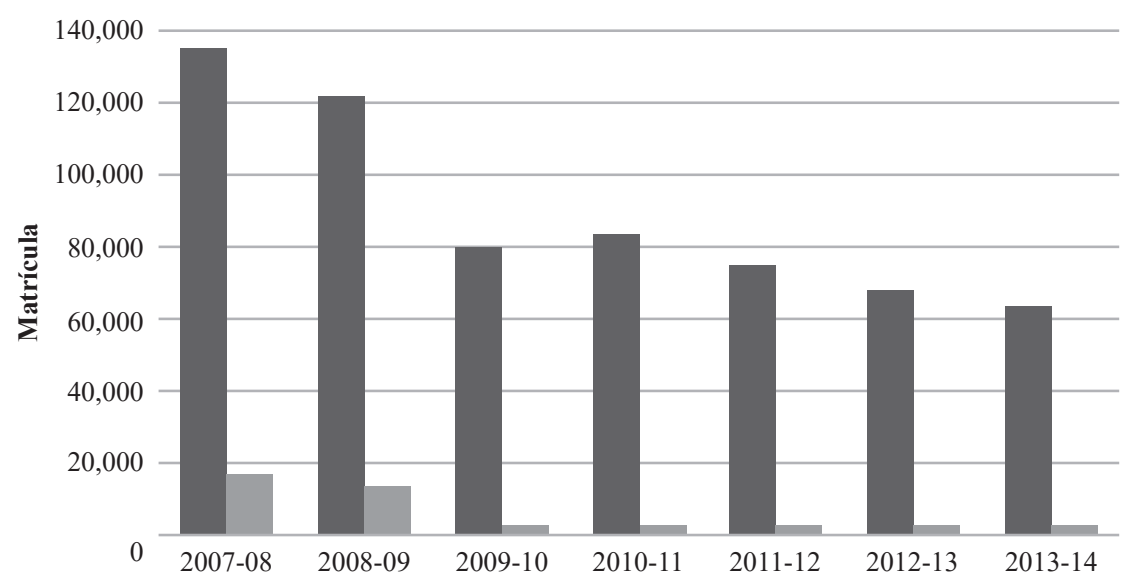

Figura 4. Estudiantes de educación migrante en California.

Fuente: Elaboración propia con datos del Departamento de Educación de California, 2017.

Al realizar un análisis histórico a la matrícula de la educación migrante en el estado de California, se observa que durante el año escolar 2007-08 la atención de estudiantes transnacionales alcanzó una cifra récord al llegar a los 135 204, de los cuales 16880 menores de edad asistían a escuelas del condado de San Diego; posteriormente, hubo un decrecimiento constante en la misma estadística hasta llegar a los 63198 alumnos a nivel estatal, es decir hubo una reducción significativa en más de 72 mil niños y adolescentes, para luego comenzar a crecer nuevamente (ver figura 4).

Si se contrastan las estadísticas educativas de California y Baja California, llegaremos a la conclusión de que la comunidad de estudiantes transnacionales en la primera es más numerosa y variada en perfiles étnicos con respecto a la segunda, por lo que la complejidad del fenómeno educativo representa un mayor desafío para el sistema escolar californiano.

\section{Conclusión}

En la región Tijuana-San Diego los problemas educativos de los estudiantes transnacionales y la desigualdad en la demostración de los aprendizajes universales, son dos fenómenos pedagógicos que cristalizan la urgente necesidad de eliminar las barreras fronterizas entre México y Estados Unidos para intervenir conjuntamente y encontrar una solución. 
Revista UniversidAd en DiÁlogo • Vol. 9, N. ${ }^{\circ}$ 1, Enero-Junio, 2019 • 63-85

ISSN 2215-2849 • EISSN: 2215-4752

URL: http://www.revistas.una.ac.cr/index.php/dialogo/index CORREO ELECTRÓNICO: universidadendialogo@una.cr

DOI: https://doi.org/10.15359/udre.9-1.4

En los nuevos enfoques del desarrollo, la educación ya no es un asunto doméstico, donde cada país es responsable de la calidad y el mejoramiento de la enseñanza; hoy en día se vuelve fundamental la creación de agendas, políticas y programas binacionales que coadyuven en la atención integral de la problemática educativa más allá de los límites fronterizos entre los países implicados, tal como sucede en otras esferas sociales (seguridad pública, migración, derechos humanos, comercio regional, turismo, etcétera).

Especialmente, el tema de los estudiantes transnacionales exige una colaboración permanente entre autoridades, directivos y docentes e investigadores de los dos países, debido a que en la región Tijuana-San Diego la matrícula transnacional de la educación obligatoria va en aumento, y puede crecer más con el retorno progresivo de migrantes mexicanos al territorio nacional, así como por los cambios en los flujos migratorios internacionales. Por lo que los sistemas educativos de México y Estados Unidos tienen el compromiso de garantizar a todos los niños y adolescentes el derecho de aprender, independientemente del origen étnico.

Para finalizar, "las políticas educativas están traspasando fronteras nacionales; de manera que se copian o trasladan de un país a otro estructuras organizativas, criterios decisionales, currículos formativos y retóricas gerenciales" (Martínez, 2008, p. 17). Entonces, para no caer en la adopción de políticas y programas educativos descontextualizados, la cooperación bilateral entre Estados UnidosMéxico, California-Baja California y San Diego-Tijuana se convierte en una herramienta útil con el propósito de instrumentar una agenda binacional de la educación para revertir la desigualdad en los aprendizajes y mejorar los procesos de formación en beneficio de los estudiantes que compartimos.

\section{Referencias bibliográficas}

Acuerdo de La Paz (1984). Departamento de Estado de los Estados Unidos de América. Recuperado de https://www.epa.gov/sites/production/ files/2015-09/documents/lapazagreement.pdf

Alcaraz, S. N. (2015). Aproximación histórica a la evaluación educativa: de la generación de la medición a la generación ecléctica. Revista Iberoamericana de Evaluación Educativa, 8(1), 11-25. Recuperado de http://www.rinace.net/riee/numeros/vol8-num1/art1.pdf

Alegría, T. (2010). Estructura de las ciudades de la frontera norte. En G. Garza \& M. Schteingart, Los grandes problemas de México. Desarrollo urbano y regional (pp. 259-304). México, D. F.: El Colegio de México. 
California Department of Education (2017). Recuperado de https://www.cde. ca.gov/

Casanova, M. A. (2012). El diseño curricular como factor de calidad educativa. Revista Iberoamericana sobre Calidad, Eficacia y Cambio en Educación. Recuperado de http://www.rinace.net/reice/numeros/ arts/vol10num4/art1.pdf

Centro de Estudios Internacionales Gilberto Bosques (2017). Panorama actual de la frontera entre México y Estados Unidos. Ciudad de México. Recuperado de http://centrogilbertobosques.senado.gob.mx/ docs/NI_FronteraMX_EEUU_010617.pdf

CEPAL (2016). Agenda 2030 y los objetivos de desarrollo sostenible. Una oportunidad para América Latina y el Caribe. Santiago: Comisión Económica para América Latina y el Caribe.

Coleman, J., Campbell, E. Q., Hobson, C., McPartland, J., Mood, A. M., Weinfeld, F. D. \& York, R. (1966). Equality of educational opportunity. Department of Health, Education \& Welfare, Office of Education, Washington, DC.

Díaz, K. y Osuna, C. (2016). Las evaluaciones estandarizadas del aprendizaje y la mejora de la calidad educativa. Revista Temas de Educación, 22(1), 131-146.

Elorriaga, M., Blancas, L. \& Ramos, A. (2015). Innovative Educational Technologies. InET Consortium Incentive Grant. Recuperado de https:// www.gob.mx/cms/uploads/attachment/file/133284/probem2015.pdf

Escudero, E. T. (2003). Desde los test hasta la investigación evaluativa actual. Un siglo, el XX, de intenso desarrollo de la evaluación en educación. Revista Electrónica de Investigación y Evaluación Educativa, 9(1). Recuperado de http://www.uv.es/relieve/v9n1/RELIEVEv9n1_1.htm

Gobierno de Baja California (2015). Segundo Informe de Gobierno. Gobierno del Estado de Baja California 2013-2019.

Gobierno de Baja California (2016). Tercer Informe de Gobierno. Educación para la Vida. Gobierno del Estado de Baja California 2013-2019.

Gobierno de Baja California (2017). Cuarto Informe de Gobierno. Gobierno del Estado de Baja California 2013-2019. 
Guillén, T. (1993). Municipal Goverment and Development in Tijuana. En N. Clement \& E. Zepeda, San Diego-Tijuana in transitions: a regional analysis. San Diego, CA: Institute for Regional Studies of the Californias.

INEE (2013). México en PISA 2012. México, D.F.: Instituto Nacional para la Evaluación de la Educación.

Jalili, N. (2015). Condiciones contemporáneas de la relación México-Estados Unidos. Hacia una movilidad estudiantil y académica. Dirección General de Relaciones Internacionales de la Secretaría de Educación Pública.

López, Y. (2015). PROBEM Baja California. XXVIII Reunión Binacional del PROBEM. México, D.F.: Sistema Educativo Estatal de Baja California.

Lorenzen, M. (2016). Migración de niñas, niños y adolescentes: antecedentes y análisis de información de la Red de Módulos y Albergues de los Sistemas DIF, 2007-2016. México D.F.: Consejo Nacional de Población.

Martínez, R. J. (2008). Globalización de los discursos de calidad y evaluación y su impacto en la identidad del profesorado. En J. L. Aróstegui \& J. B. Martínez, Globalización, posmodernidad y educación. La calidad como coartada neoliberal (pp. 15-46). Madrid: Ediciones Akal.

National Center for Education Statistics (2013). Trends in International Mathematics and Science Study. Recuperado de: https://nces.ed.gov/ timss/index.asp

National Center for Education Statistics (2016). State Education Data Profiles 2015-2016. Recuperado de https://nces.ed.gov/programs/stateprofiles/ index.asp

Organización para la Cooperación y el Desarrollo Económico (2016). PISA 2015 , resultados clave. Recuperado de https://www.oecd.org/pisa/

Osuna, J., Miramontes, M. A. y Villaseñor, M. G. (2018). Los estudiantes transnacionales en México: actores olvidados en la agenda pública de la educación. VI Congreso Nacional de Ciencias Sociales. Consejo Mexicano de Ciencias Sociales, San Luis Potosí.

PROBEM México (2015). Alcances y metas del Programa Binacional de Educación Migrante en México. Secretaría de Educación Pública/ Secretaría de Relaciones Exteriores. 
Rocha, D. y Orraca, P. (2018). Estudiantes de educación superior transfronterizos: residir en México y estudiar en Estados Unidos. Revista Frontera Norte, 30(59).

Serrano, C. y Jaramillo, M. (2017). Anuario de migración y remesas. México 2017. Ciudad de México: Fundación BBVA Bancomer y Consejo Nacional de Población.

Sistema Educativo Estatal (2015). Programa de educación de Baja California 2015-2019. Sistema Educativo Estatal de Baja California.

UNESCO(2013).Haciaunaprendizajeuniversal.Recomendaciones dela Comisión Especial sobre Métricas de los Aprendizajes. Montreal y Washington D.C.: UNESCO y Centro de Educación Universal de Brookings.

Weeks, J. (1993). The changing demographic structure of the San Diego region. En C. Norris \& E. Zepeda, San Diego-Tijuana in transitions: a regional analysis. San Diego, CA: Institute for Regional Studies of the Californias.

Zúñiga, V. (2008). Reflexiones sobre el fracaso escolar y los alumnos transnacionales en las escuelas de México. En V. Zúñiga, E. Hamann y J. Sánchez, Alumnos transnacionales. Escuelas mexicanas frente a la globalización (pp. 61-77). México, D.F.: Secretaría de Educación Pública.

Zúñiga, V. (2013). Migrantes internacionales en las escuelas mexicanas: desafíos actuales y futuros de política. Revista Sinéctica, (40). Recuperado de http://www.redalyc.org/articulo.oa?id=99827467009 